\title{
ON CHARWAT'S THEORY OF MOTION OF TRACERS IN PLANAR VORTEX FLOWS
}

\author{
R. B. LEIPNIK'
}

(Received 4 January 1982; revised 1 July 1982)

\begin{abstract}
The motion of small, near neutrally buoyant tracers in vortex flows of several types is obtained on the basis of Charwat's mathematical model, which is highly non-linear.

The solution method in the non-degenerate case expresses the squared orbital radius $r^{2}$ as a product $A A^{*}$, where the complex number $A$ satisfies a second-order linear differential 'factor equation', generally with variable coefficients. The angular coordinate is expressed in terms of $\log \left(A^{*} / A\right)$. Solid-type rotation and sinusoidally perturbed solid-type rotation correspond respectively to constant coefficients and sinusoidal coefficients. The former exactly yields a scalloped spiral tracer motion; the latter yields unstable tracer motion as $t \rightarrow \infty$ except when the perturbing frequency and amplitude are rather specially related to the flow and tracer parameters. Free vortex motion is somewhat degenerate for this solution method but can be partially analyzed in terms of solutions of a generalized Emden-Fowler equation. The method can be used for other planar flow problems with a symmetry axis.
\end{abstract}

\section{Introduction}

Visualization of fluid flows by following the motion of tracers-small, neutrally buoyant particles-has long been practiced. The use of laser holography has enhanced the feasibility of this approach to the more complex flows, including vortex flows. Charwat [1] has discussed the experimental and theoretical aspects of tracer motion in planar vortex flows. He obtained many properties of the motion (expressed in polar coordinates) for two cases: "solid-body" vortex flow

\footnotetext{
${ }^{1}$ Department of Mathematical Physics, University of Adelaide, G.P.O. Box 498, Adelaide, S.A. 5001. Permanent Address: Department of Mathematics, University of California, Santa Barbara, California 93106, U.S.A.

(c) Copyright Australian Mathematical Society 1983. Serial-fee code 0334-2700/83.
} 
and "free" vortex flow. However, he did not obtain complete solutions, in either case.

Rather similar (but much simpler) non-linear equations appear in the theory of the motion of a charged particle, or a pair of charged particles, in a time-dependent solenoid (cylindrically symmetric magnetic field). These were solved $[3,6]$ by a method based on a Riccati equation and complex numbers. An adaptation of this method is used in this paper. In Section 2, the solenoid case is reviewed for insight into the method. Section 3 contains the Charwat equations and the first stage of the solution.

Section 4 completes the solution in the non-degenerate case, where "separation of powers" succeeds. The solid-body vortex is a special example. In Section 5, a complete explicit solution in elementary terms is obtained both for the radial and angular transient behaviour. Charwat's qualitative results are supported entirely. In addition, a transient damped periodic scalloping, with all harmonics present, of both the radial and angular coordinates is noted.

Section 6 takes up the degenerate case and shows that a generalization of the non-degenerate technique is necessary. The free vortex motion is easily seen to be degenerate and reduces to an equation of Painlevé studied by Gambier (Ince [4] and Fowler [2]). For a special initial condition, the equation reduces further to a form discussed in Section 2.

Section 7 goes beyond the two cases introduced by Charwat to the situation of a periodically perturbed flow. In the special case of a single harmonic, the reduced Charwat equations specialize to damped Mathieu equations and hence, by a further transformation, to undamped Mathieu equations. As is well-known, Mathieu equations possess an infinite family of parametrical 'islands' of stability, set in a 'sea' of instability. The motion of the tracers will in general be chaotic, unless the perturbation frequency $\nu$ and amplitude $h$ are suitably related to quantities appearing in the unperturbed motion. Conditions on $\nu$, so that the solutions fall in the first stability island, are developed.

\section{Motion of particles in a time-dependent solenoid}

If $e, m$ are the charge and mass of a pair of particles acting under a potential $\phi(r)$ in a uniform but time-dependent magnetic field $\mathbf{B}$, then

$$
m d \rho / d t=e(\rho \times \mathbf{B}) / c-2 \phi^{\prime}(r) \mathbf{r} / r,
$$

where $\rho$ is the relative velocity and $\mathbf{r}$ the relative position. If the $x_{3}$-axis is parallel to $\mathbf{B}$, and the $x_{2}$-axis is normal to the initial relative position $\mathbf{r}_{\mathbf{0}}$, the equation (1) is simplified in rotating coordinates $x_{1}^{\prime}=y_{1}, x_{2}^{\prime}=y_{2}$, where the rotation velocity is 
the Larmor velocity $\omega / 2=e|\mathbf{B}| /(2 m c)$. The result is

$$
\begin{aligned}
d \eta / d t & =d^{2} \mathbf{y} / d t^{2}=-\left(\omega^{2} / 4+2 \phi^{\prime}(r) / m r\right) / y, \\
d^{2} r_{3} / d t^{2} & =d \rho / d t=-2 \phi^{\prime}(r) r_{3} / m r .
\end{aligned}
$$

The first equation has the invariant $(\mathbf{y} \times \boldsymbol{\eta}) \cdot \mathbf{B} / B=y_{1} \eta_{2}-y_{2} \eta_{1}=C$. Introducing polar coordinates $y_{1}=y \cos \theta, y_{2}=y \sin \theta$, we find

$$
y^{2} \dot{\theta}=C, \quad \ddot{y}+\left(2 \phi^{\prime}(r) / r+\omega^{2} / 4\right) y=C^{2} / y^{3} .
$$

If we replace the spatial dependence of $\phi^{\prime}(r) / r$ by a time dependence, through an iterative procedure [3], we can reduce the problem to

$$
y^{2} \dot{\theta}=C, \quad \ddot{y}+\Omega^{2} y / 4=C^{2} / y^{3},
$$

where $C$ is a constant and $\Omega$ is time-dependent.

The Ansatz

$$
\dot{y}=\dot{A} y / A+i C / y
$$

yields

$$
\begin{aligned}
\ddot{y} & =\left(\ddot{A} / A-\dot{A}^{2} / A^{2}\right) y+\left(\dot{A} / A-i C / y^{2}\right) \dot{y} \\
& =\left(\ddot{A} / A-\dot{A}^{2} / A^{2}\right) y+\left(\dot{A} / A-i C / y^{2}\right)(\dot{A} y / A+i C / y)=\ddot{A} y / A+C^{2} / y^{3} .
\end{aligned}
$$

Thus (4) is satisfied, provided that

$$
\ddot{A}+\Omega^{2} A / 4=0 \text {, }
$$

a differential equation for $A$ which resembles (4) except that

(a) the non-linearity on the right is removed,

(b) $A$ is not necessarily real-valued.

Now from (4) and (5), $\dot{y}=\dot{A} y / A+i y^{2} \dot{\theta} / y=(\dot{A} / A+i \dot{\theta}) y$, so $\dot{y} / y=\dot{A} / A+$ $i \dot{\theta}$, which integrates to $y=K A e^{i \theta}$. At $\theta=0, y=A$ so $K=1$ and

$$
y=A e^{i \theta} \text {. }
$$

Since $\theta$ and $y$ are both real,

$$
y^{2}=A A^{*}, \quad e^{i \theta}=\left(A^{*} / A\right)^{1 / 2} .
$$

Thus the complex solution of (7) readily yields the real quantities $y$ and $\theta$. The non-linear equation has been exactly solved in terms of a simple non-linear function of the solution of a linear differential equation. This is true whether $\Omega$ is constant or time-dependent. The result has implications for diffusion and conductivity of plasma in strong magnetic fields, but it is the mathematical technique which is of interest now. 


\section{Equations of motion of tracers}

In the notation of Charwat [1], the equations of motion of a tracer particle in polar coordinates are

$$
\begin{aligned}
\ddot{r}-r \omega^{2} & =-S(\partial P / \partial r)_{f} / \rho_{f}+S B\left(u_{f}-r\right), \\
r \dot{\omega}+2 \omega \dot{r} & =-S(\partial P / \partial \theta)_{f} / r \rho_{f}+S B\left(v_{f}-\omega r\right),
\end{aligned}
$$

where

$$
S=\rho_{f} / \rho_{p}, \quad B=9 v_{f} /\left(2 a^{2}\right) .
$$

Here gravity is neglected, and the gradient terms are due to pressure on the particle surface [12, 4]. The drag force is taken to be Stokesian. If the specific pressure gradients are radially symmetrical,

$$
(\partial P / \partial \theta)_{f} / \rho_{f}=\Theta, \quad(\partial P / \partial r)_{f} / \rho_{f}=R,
$$

where $R, \Theta$ depend on $r$ (and on $t$, since this does not invalidate the method to be used). Similarly, we can allow $u_{f}$ and $v_{f}$ to depend on $t$ to a certain extent. Hence the equations become

$$
\begin{gathered}
\ddot{r}-r \omega^{2}=-S R(r)+S B\left(u_{f}-\dot{r}\right), \\
r \dot{\omega}+2 \omega \dot{r}=-S \Theta(r) / r+S B\left(v_{f}-\omega r\right) .
\end{gathered}
$$

Multiplication of (14) by $r$ yields

$$
\left(r^{2} \omega\right)^{\cdot}=r^{2} \dot{\omega}+2 \omega r \dot{r}=-S \Theta(r, t)+S B\left(v_{f} r-r^{2} \omega\right),
$$

a first-order differential equation in $r^{2} \omega$.

Integration from time $t_{0}=0$ yields

$$
r^{2} \omega=r_{0}^{2} \omega_{0} e^{-B S t}+S e^{-B S t} \int_{0}^{t}\left[B v_{f}\left(t_{1}\right) r\left(t_{1}\right)-\Theta\left(r\left(t_{1}\right), t_{1}\right)\right] e^{B S t_{1}} d t_{1}
$$

Hence

$$
r \omega^{2}=r^{-3} e^{-2 B S t}\left(r_{0}^{2} \omega_{0}+S \int_{0}^{t}\left(B v_{f} r-\Theta\right) e^{B S t_{1}} d t_{1}\right)^{2}
$$

Elimination of $\omega$ from (13) with the help of (17) yields

$$
\begin{aligned}
\ddot{r}+S B \dot{r}= & r^{-3} e^{-2 B S t}\left(r_{0}^{2} \omega_{0}+S \int_{0}^{t}\left(B v_{f} r-\Theta(r)\right) e^{B S t_{1}} d t_{1}\right)^{2} \\
& -S R(r)+S B u_{f},
\end{aligned}
$$

an integro-differential equation for $r$. Note the resemblance to (4). Equations (15)-(18), not used by Charwat, provide the point of departure of this paper. 


\section{The solution method}

We try the Ansatz

$$
\dot{r}=F(t) r+i\left(d(t)+e(t) \int_{0}^{t} \chi\left(r\left(t_{1}\right), t_{1}\right) f\left(t_{1}\right) d t_{1}\right) / r,
$$

where $d, e, \chi, f, F$ are to be determined. The coefficient of $1 / r$ in (19) is patterned after the coefficient of $1 / r^{3}$ in (18).

If $F, d, e, f, \chi$ are well-behaved, then

$$
\begin{aligned}
\ddot{r}= & i\left(\dot{d}+\dot{e} \int_{0}^{t} \chi\left(r\left(t_{1}\right)\right) f\left(t_{1}\right) d t_{1}+e(t) f(t) \chi(r)\right) / r \\
& -i\left(d+e \int_{0}^{t} \chi\left(r\left(t_{1}\right)\right) f\left(t_{1}\right) d t_{1}\right) \dot{r} / r^{2}+\dot{F} r+F \dot{r} .
\end{aligned}
$$

Substitution for $\dot{r}$ from (19) yields

$$
\begin{aligned}
r+S B \dot{r}= & \left(d+e \int_{0}^{t} \chi f d t_{1}\right)^{2} / r^{3} \\
& +i\left[S B\left(d+e \int_{0}^{t} \chi f d t_{1}\right)+\dot{d}+\dot{e} \int_{0}^{t} \chi f d t_{1}+e \chi f\right] / r \\
& +\left[F^{2}+\dot{F}+S B F\right] r .
\end{aligned}
$$

Comparison with (18) yields, if $S\left(B v_{f}-R\right) \neq r^{-3} N(t)$ for some $N(t)$,

$$
d+e \int_{0}^{t} \chi f d t_{1}=e^{-B S t}\left(r_{0}^{2} \omega_{0}+S \int_{0}^{t}\left(B v_{f} r-\Theta\right) e^{B S t_{1}} d t_{1}\right)
$$

for the coefficient of $r^{-3}$. The case $S\left(B v_{f}-R\right)=r^{3} N(t)$ is degenerate as the "separation of powers" fails.

Hence, from (22),

$$
\begin{aligned}
& d=r_{0}^{2} \omega_{0} e^{-B S t}, \quad e(t)=S e^{-B s t}, \\
& f=e^{B S t}, \quad \chi(r, t)=B v_{f}(t) r-\Theta(r, t),
\end{aligned}
$$

with no restrictions on $v_{f}$ or $\Theta$ (as yet); a surprising result.

Since $\dot{d}+S B d=\dot{e}+S B e=0$, the coefficient of $r^{-1}$ in (21) reduces to ie $\chi f=$ $i S \chi$. Thus, from (18),

$$
S\left(B u_{f}-R\right)=i S\left(B v_{f} r-\Theta\right) / r+\left(F^{2}+\dot{F}+S B F\right) r .
$$

Now $F$ is independent of $r$, but not necessarily real-valued, so we write

$$
F^{2}+\dot{F}+S B F=S G(t)=S\left(G_{1}+i G_{2}\right),
$$


where $G_{1}, G_{2}$ are undetermined. But from (24), on equating real and imaginary parts, we find

$$
\begin{gathered}
B u_{f}-R=r G_{1}(t), \\
B v_{f} r-\Theta=-r^{2} G_{2}(t)=\chi .
\end{gathered}
$$

Thus we can consider pairs $u_{f}(t), R(r, t)$ and $v_{f}(t), \Theta(r, t)$ which satisfy the conditions (26) and (27) for reasonably general $G_{i}(t)$ and $G_{2}(t)$.

\section{Reduction to linear differential equations}

A Riccati equation of the type (25) can be resolved by the Bernoulli substitution $F=\dot{A} / A$, resulting in

$$
\ddot{A}+S B \dot{A}=S G(t) A .
$$

Here $A$ is analogous to the $A$ employed in Section 1 . Note as before that the non-linear equation has been replaced by a linear equation (28). The simple substitution $A=W e^{-B S t / 2}$ yields the equation

$$
\ddot{W}-(S B)^{2} W / 4=S e^{B S t / 2} G(t) W .
$$

Obviously, the full resources of classical variable coefficient theory can be applied to this problem, after $G(t)$ is chosen. Once $A$ (or $W$ ) is determined, then $F$ is known. This brings us back to the Ansatz (19).

Since $\chi(r, t)=B v_{f} r-\Theta=-r^{2} G_{2}(t)$ from (27), we have

$$
\dot{r}=F r+i e^{-B S t}\left(r_{0}^{2} \omega_{0}-S \int_{0}^{t} r^{2} G_{2} e^{B S t_{1}} d t_{1}\right) / r .
$$

The squared distance variable $\delta=r^{2}$ yields the equation

$$
\dot{\delta}=2 F \delta+2 i e^{-B S t}\left(\delta_{0} \omega_{0}-S \int_{0}^{t} \delta G_{2} e^{B S t_{1}} d t_{1}\right) .
$$

Note that setting $t=0$ in (31) yields $\dot{\delta}_{0}=2 F(0) \delta_{0}+2 i \delta_{0} \omega_{0}$. Since $\delta_{0}, \omega_{0}$ and $\dot{\delta}_{0}$ are real, we see that $F(0)$ is complex-valued as expected. Also, from (31),

$$
\begin{aligned}
\ddot{\delta}= & 2 \dot{F} \delta+2 F \dot{\delta}+2 i e^{-B S t}\left(-S \delta G_{2} e^{B S t}\right) \\
& -2 i B S e^{-B S t}\left(\delta_{0} \omega_{0}-S \int_{0}^{t} \delta G_{2} e^{-B S t_{1}} d t_{1}\right) .
\end{aligned}
$$

Elimination of the integral term between (31) and (32) yields

$$
\ddot{\delta}+(B S-2 F) \dot{\delta}=2\left(\dot{F}+B S F-i S G_{2}\right) \delta,
$$

another linear second-order equation with variable coefficients. Since $\dot{F}+B S F=$ $S G_{1}+i S G_{2}-F^{2}$, the coefficient of $\delta$ can also be written as $2\left(S G_{1}-F^{2}\right)$. 
Since $F$ need not be simple, even when $G(t)$ is simply chosen (or, alternatively, when $R, \theta, u_{f}, v_{f}$ are simple), the solution of (33) will not be pleasant. Again, however, the resources of the classical theory are available. Recall that, just as real coefficients do not imply real solutions, so also complex coefficients do not forbid real solutions (if the coeffficients are suitably related). Fortunately, the solid-type rotation case of Charwat [1] can be completely solved from the above, as shown in the next section.

\section{Solid-type rotation}

The case of solid-type rotation, qualitatively resolved by Charwat [1], is defined by

$$
\begin{aligned}
& u_{f}=0, \quad v_{f}=\omega_{f} r=K_{s} r, \\
& \Theta=(\partial p / \partial \theta)_{f} / \rho f=0, \quad R=(\partial p / \partial r)_{f} / \rho_{f}=\omega_{f}^{2} r=K_{s}^{2} r .
\end{aligned}
$$

Thus

$$
\begin{gathered}
B u_{f}-R=-K_{s}^{2} r=r G_{1}(t), \quad G_{1}(t)=-K_{s}^{2}, \\
B v_{f} r-\Theta=B K_{s} r^{2}=-r^{2} G_{2}(t), \quad G_{2}(t)=-B K_{s} .
\end{gathered}
$$

This fits into the standard case, and can be completely solved. From (25), we have

$$
\dot{F}+F^{2}+S B F=S K_{s}\left(-K_{s}-i B\right)=\text { constant. }
$$

Setting $F=\dot{A} / A$ yields, as in (28), the linear system

$$
\ddot{A}+S B \dot{A}+S K_{s}\left(K_{s}+i B\right) A=0 .
$$

Clearly $(S B)^{2} \neq 4 S K_{s}\left(K_{s}+i B\right)$, unless $B=0$ and $S K_{s}=0$, so the solution of (37) takes the form

$$
A=A_{1} e^{p_{1} t}+A_{2} e^{p_{2} t}
$$

where

$$
\begin{aligned}
p_{1}, p_{2} & =\left(-S B \pm \sqrt{(S B)^{2}-4 S K_{s}\left(K_{s}+i B\right)}\right) / 2 \\
& =-S B / 2 \pm(\alpha+i \beta),
\end{aligned}
$$

where $\alpha, \beta$ are real.

Note that Charwat's "more practical" case $S B / 2>K_{s} \sqrt{S}$ exactly corresponds to the positivity of real part of the radicand of (38).

It follows that

$$
F=\left(p_{1} A_{1} e^{p_{1} t}+p_{2} A_{2} e^{p_{2} t}\right) /\left(A_{1} e^{p_{1} t}+A_{2} e^{p_{2} t}\right)
$$


and $\delta=r^{2}$ satisfies the equation

$$
\ddot{\delta}+(B S-2 F) \dot{\delta}=2\left(\dot{F}+B S F-i S B K_{s}\right) \delta,
$$

a linear equation with complicated variable coefficients.

This equation is reminiscent of (37), so let us try a solution of the form

$$
\delta=A w \text {, }
$$

where $F=\dot{A} / A$.

Substitution of (41) into (40) and multiplication by $A$ yields

$$
\begin{aligned}
A(A w)^{*}+ & A(B S-2 \dot{A} / A)(A w)^{\circ} \\
& -2 A^{2}\left(\ddot{A} / A-\dot{A}^{2} / A^{2}+B S \dot{A} / A-i S B K_{s}\right) w=0 .
\end{aligned}
$$

Collecting terms in $w, \dot{w}, \ddot{w}$ we find

$$
\begin{aligned}
0= & A^{2} \ddot{w}+\left(B S A^{2}-2 A \dot{A}+2 A \dot{A}\right) \dot{w} \\
& +\left(A \ddot{A}+B S A \dot{A}-2 \dot{A}^{2}-2 A \ddot{A}+2 \dot{A}^{2}-2 B S A \dot{A}+2 A^{2} i S B K_{s}\right) w .
\end{aligned}
$$

On substitution of $\ddot{A}$ from (37), (43) reduces exactly to

$$
A^{2}\left[\ddot{w}+B S \dot{w}+\left(S K_{s}^{2}-i S B K_{s}\right) w\right]=0,
$$

so that $w$ satisfies

$$
\ddot{w}+B S \dot{w}+\left(S K_{s}\right)\left(K_{s}-i B\right) w=0,
$$

whose form closely resembles (37).

In fact, the solution is $w=w_{1} e^{q_{1} t}+w_{2} e^{q_{2} t}$ where

$$
\begin{aligned}
q_{1}, q_{2} & =\left(-S B \pm \sqrt{(S B)^{2}-4 S K_{s}\left(K_{s}-i B\right)}\right) / 2 \\
& =-S B / 2 \pm(\alpha-i \beta),
\end{aligned}
$$

where $\alpha, \beta$ appeared already in (38).

If $S K_{s} B \neq 0$, then $\alpha \neq 0, \beta \neq 0$. Take $\alpha>0$ without loss of generality. Then

$$
\begin{aligned}
\delta & =A w=\left(A_{1} e^{p_{1} t}+A_{2} e^{p_{2} t}\right)\left(w_{1} e^{q_{1} t}+w_{2} e^{q_{2} t}\right) \\
& =e^{-B S t}\left(A_{1} w_{1} e^{2 \alpha t}+A_{2} w_{2} e^{-2 \alpha t}+A_{1} w_{2} e^{2 \imath \beta t}+A_{2} w_{1} e^{-2 \imath \beta t}\right) .
\end{aligned}
$$

Since $\delta=r^{2}>0$, it follows that

$$
\left(A_{1} w_{1}\right)^{*}=A_{1} w_{1}>0, \quad\left(A_{2} w_{2}\right)^{*}=A_{2} w_{2}>0, \quad\left(A_{1} w_{2}\right)^{*}=A_{2} w_{1} .
$$

Setting

$$
\begin{aligned}
& A_{1}=\left|A_{1}\right| e^{i \theta_{1}}, \quad A_{2}=\left|A_{2}\right| e^{i \theta_{2}}, \\
& \delta_{1}=A_{1} w_{1}, \quad \delta_{2}=A_{2} w_{2},
\end{aligned}
$$


we find

$$
\delta=r^{2}=e^{-S B t}\left[r_{1}^{2} e^{2 \alpha t}+r_{2}^{2} e^{-2 \alpha t}+2 r_{1} r_{2} \cos \left(\theta_{1}-\theta_{2}+2 \beta t\right)\right] .
$$

This defines a "scalloped" spiral motion, with a fixed scalloping frequency $2 \beta$, and with an exponentially damped scalloping amplitude. Three cases are obvious:

(i) $2 \alpha=S B, \delta=r^{2}=r_{1}^{2}+e^{-S B t}\left(r_{2}^{2} e^{-2 \alpha t}+2 r_{1} r_{2} \cos \left(\theta_{1}-\theta_{2}+2 \beta t\right)\right)$. Here the radius decreases in an oscillatory fashion from

$$
\bar{r}_{0}=\left(r_{1}^{2}+r_{2}^{2}+2 r_{1} r_{2} \cos \left(\theta_{1}-\theta_{2}\right)\right)^{1 / 2}
$$

at $t=0$ to $\bar{r}_{\infty}=r_{1}$ at $t=\infty$, yielding a stable limit cycle.

(ii) $2 \alpha<S B, r \rightarrow 0$ as $t \rightarrow \infty, r \rightarrow \infty$ as $t \rightarrow-\infty$, also stable as $t \rightarrow \infty$.

(iii) $2 \alpha>S B, r \rightarrow \infty$ as $t \rightarrow \infty, r \rightarrow 0$ as $t \rightarrow-\infty$, i.e. instability.

More detailed information can be gleaned, following Charwat, from the relative radial rate $q=\dot{r} / r=\dot{\delta} / 2 \delta$ and the angular velocity

$$
\begin{aligned}
\omega & =r^{-2} e^{-B S t}\left(r_{0}^{2} \omega_{0}+S \int_{0}^{t}\left(B v_{f} r-\Theta\right) e^{B S t_{1}} d t_{1}\right) \\
& =\left(\delta e^{B S t}\right)^{-1}\left(r_{0}^{2} \omega_{0}+S \int_{0}^{t} B K_{s} \delta\left(t_{1}\right) \cdot e^{B S t_{1}} d t_{1}\right) .
\end{aligned}
$$

But clearly

$$
\begin{aligned}
\int_{0}^{t} \delta\left(t_{1}\right) e^{B S t_{1}} d t_{1}= & r_{1}^{2}\left(e^{2 \alpha t}-1\right) / 2 \alpha-r_{2}^{2}\left(e^{-2 \alpha t}-1\right) / 2 \alpha \\
& +\left(2 r_{1} r_{2} / 2 \beta\right)\left[\sin \left(\theta_{1}-\theta_{2}+2 \beta t\right)-\sin \left(\theta_{1}-\theta_{2}\right)\right] .
\end{aligned}
$$

As $t \rightarrow \infty, \omega \rightarrow r_{1}^{2} S B K_{s} /\left(2 \alpha r_{1}^{2}\right)=S B K_{s} / 2 \alpha$, and as $t \rightarrow-\infty$,

$$
\omega \rightarrow-r_{2}^{2} S B K_{s} /\left(2 \alpha r_{2}^{2}\right)=-S B K_{s} / 2 \alpha .
$$

Thus $\omega$ is the ratio of two damped oscillatory expressions with the same scalloping frequency $2 \beta$. Similarly, $q$ is a ratio of two damped oscillatory expressions and tends to a limit as $t \rightarrow \infty$ and as $t \rightarrow-\infty$.

In fact,

$$
q=\frac{1}{2} \frac{d}{d t}\left[\log \left(r_{1}^{2} e^{2 \alpha t}+r_{2}^{2} e^{-2 \alpha t}+2 r_{1} r_{2} \cos \left(\theta_{1}-\theta_{2}+2 \beta t\right)\right)\right]-B S / 2,
$$

so that

$$
q \rightarrow \alpha-B S / 2 \quad \text { as } t \rightarrow-\infty, \quad q \rightarrow-\alpha-B S / 2 \quad \text { as } t \rightarrow \infty .
$$

Explicitly,

$$
\begin{aligned}
\alpha, \beta & =\sqrt{(U \pm V) / 8} \\
U^{2} & =\left((S B)^{2}-4 S K_{s}^{2}\right)^{2}+16\left(S B K_{s}\right)^{2} \\
V^{2} & =(S B)^{2}-4 S K_{s}^{2}
\end{aligned}
$$


Repeated squaring and rearranging shows that $S B / 2 \gtreqless \alpha$ according as $S \gtreqless 1$, so that the three cases (i), (ii), (iii) can be recategorized as
(i) $S=1, \quad r \rightarrow r_{1}, \quad \omega \rightarrow K_{s}, \quad q \rightarrow 0$ as $t \rightarrow \infty$,
(ii) $S>1, \quad r \rightarrow 0, \omega \rightarrow S B K_{s} / 2 \alpha>K_{s}, \quad q \rightarrow \alpha-S B / 2<0 \quad$ as $t \rightarrow \infty$,
(iii) $S<1, \quad r \rightarrow \infty, \omega \rightarrow S B K_{s} / 2 \alpha<K_{s}, \quad q \rightarrow \alpha-S B / 2>0$ as $t \rightarrow \infty$.

Physically, the asymptotic results are obvious, but the transient scalloping motion is a little surprising.

\section{Degeneracy}

When $S\left(B u_{f}-R\right)=r^{-3} N(t)$, the reduction of Section 4 fails and the seemingly simpler equation

$$
\ddot{r}+B S r=r^{-3} N(t)+e^{-2 B S t}\left(r_{0}^{2} \omega_{0}+S \int_{0}^{t}\left(B v_{f} r-\Theta\right) e^{B s t_{1}} d t_{1}\right)^{2},
$$

results. The Ansatz (19) now fails. The more complex Ansatz

$$
\dot{r}=F_{-1} r+i(d(t)+e(t) \sigma(r, t)) / r+\sum_{K=2}^{\infty} F_{K}(t) r^{-K}
$$

leads to a sequence of differential equations for $F_{-1}, F_{2}, \ldots$ All but the first are linear, but with variable coefficients, depending on the previous $F$ 's. The free vortex case is a specially simple degenerate case, and can be carried somewhat further.

\section{Free Vortex}

For the free vortex,

$$
u_{f}=0, \quad v_{f}=K_{p} / r, \quad \Theta=0, \quad R=K_{p}^{2} / r^{3},
$$

so that

$$
\begin{aligned}
S\left(B u_{f}-R\right) & =-S K_{p}^{2} / r^{3}=N(t) / r^{3}, \\
B v_{f} r-\Theta & =B K_{p}=\text { constant }
\end{aligned}
$$

which is a degenerate case. 
Thus (55) reduces to

$$
\begin{gathered}
\ddot{r}+B S \dot{r}=r^{-3} H(t), \\
H(t)=H_{0}+H_{1} e^{-B S t}+H_{2} e^{-2 B S t}, \\
H_{0}=K_{p}^{2}(1-S), \quad H_{1}=2 K_{p}\left(r_{0}^{2} \omega_{0}-K_{p}\right), \\
H_{2}=\left(r_{0}^{2} \omega_{0}-K_{p}\right)^{2} .
\end{gathered}
$$

In the variable $\delta=r^{2}$, (59) becomes

$$
\ddot{\delta}-\dot{\delta}^{2} / 2 \delta+B S \dot{\delta}=2 H(t) / \delta,
$$

an equation of the classic Painlevé-Gambier type, discussed in Ince [4]. Unfortunately, with the above choice of $H(t)$, it is not one of the 50 types which have a known first integral in closed form, many of which define new (Painlevé-Gambier) transcendants.

However, if either ( $\alpha) S=1$ (neutral buoyancy) or $(\beta) K_{p}=r_{0}^{2} \omega_{0}$ or $(\gamma)$ $K_{p}=0$, which yield respectively $(\alpha) H_{0}=0,(\beta) H_{1}=H_{2}=0$, or $(\gamma) H_{0}=H_{1}$ $=0$, the equation (59) reduces to an Emden-Fowler equation, repeatedly studied by Fowler [2]. This follows from using an exponential substitution $\tau=e^{-B S t}$ in (59). The result, if $G(\tau)=r(t)$, is

$$
G^{3}(\tau) G^{\prime \prime}(\tau)=(1 / B S)\left(H_{0} / \tau+H_{1}+H_{2} \tau\right) .
$$

With the help of (59), the cases $(\alpha),(\beta),(\gamma)$ can each be put in the Fowler form

$$
\theta^{\prime \prime}(x)+x^{\sigma} \theta^{n}=0,
$$

with $n=-3$ and $\sigma=-1$ (case $(\alpha)), \sigma=0$ (case $(\beta))$ and $\sigma=1$ (case $(\gamma)$ ). Fowler's own discussion is only for $n>0$ but the analysis is similar for $n \leqslant 0$.

Note that

$$
\theta_{0}(x)= \pm[\chi(1-\chi)]^{-1 / 4} x^{x}
$$

is a special solution, where $\chi=(\sigma+2) / 4$, provided that $0<\chi<1$.

Hence write $\theta=\theta_{0}(x) v(x)$ and obtain the equation

$$
x^{2} v^{\prime \prime}+2 \chi x v^{\prime}-\chi(1-\chi)\left(v-v^{-3}\right)=0 .
$$

The substitution $y=\log x, u(y)=v(x)$ yields

$$
u^{\prime \prime}+(2 \chi-1) u^{\prime}-\left(\chi-\chi^{2}\right)\left(u-u^{n}\right)=0 .
$$

The results are, according to Fowler, similar in the three cases $(\alpha) \chi=\frac{1}{4},(\beta)$ $\chi=\frac{1}{2},(\gamma) \chi=\frac{3}{4}$. They are markedly simpler when $\chi=\frac{1}{2}$, since then the equation (65) reduces to

$$
u^{\prime \prime}-u / 4=-1 / 4 u^{3},
$$

which is of the type solved in Section 2, if we take $\Omega=i$ and $C=i / 2$. In all cases, the results are sensitive to the initial conditions. 


\section{Factorization and sinusoidal perturbation}

The tracer motions in solid-type rotation involve only $G(t)=$ constant and thus could have been deduced by methods of greater simplicity, based on writing the Charwat equations in the plane rectangular form

$$
\ddot{\mathbf{x}}+S B \dot{\mathbf{x}}-S \mathbf{x} G_{1}(t)=S(\mathbf{x} \times \mathbf{k}) G_{2}(t)
$$

and so in the complex form

$$
\ddot{z}+S B \dot{z}-S z G(t)=0,
$$

where $G=G_{1}+i G_{2}, z=x_{1}+i x_{2}$, and $\mathbf{k}$ is normal to the $\left(x_{1}, x_{2}\right)$ plane.

When $G(t)$ is non-constant, the rectangular form is somewhat less effective, relative to the polar form, as is well known from the theory of Prüfer transformations.

The factorization $\delta=A w$ carried out in the case $G(t)=$ constant in Section 5 is valid generally. To see this, note that if $\delta=A w$, and $F=\dot{A} / A$, then by (33)

$$
\begin{aligned}
0= & (A w)+(B S-2 \dot{A} / A)(A w)-2 A w\left((\dot{A} / A)+B S \dot{A} / A-i S G_{2}\right) \\
= & \ddot{A} w+2 \dot{A} \dot{w}+A \ddot{w}+B S(\dot{A} w+A \dot{w})-2\left(\dot{A}^{2} / A\right) w-2 \dot{A} \dot{w} \\
& -2 \ddot{A} w+2\left(\dot{A}^{2} / A\right) w-2 B S \dot{A} w+2 i A w S G_{2} \\
= & A \ddot{w}-\ddot{A} w-B S \dot{A} w+B S A \dot{w}+2 i A w S G_{2} \\
= & A w\left(\ddot{w} / w+B S \dot{w} / w+i S G_{2}-S G_{1}\right) \\
& -A w\left(\ddot{A} / A+B S \dot{A} / A-i S G_{2}-S G_{1}\right) .
\end{aligned}
$$

Substitution of (28) yields

$$
0=A\left(\ddot{w}+B S w-S G^{*}(t) w\right) .
$$

The initial condition $\dot{\delta_{0}}=2\left(F(0)+i \omega_{0}\right) \delta_{0}$ from (31) et seq. yields the relation

$$
\dot{w}_{0} w_{0}-\dot{A}(0) / A(0)=2 i \omega_{0} .
$$

The problem remains of solving

$$
\ddot{A}+B S \dot{A}=S G(t) A,
$$

(and so its conjugate for $w$ ) under the relevant assumptions on $G(t)$. An interesting non-trivial case is $G(t)$ periodic, a Hill condition, of which only $G(t)=g_{1}+i g_{2}+h_{1} \cos \nu t+i h_{2} \sin \nu t$ yields well-understood behaviour. This can be viewed as a 'parametric' perturbation of solid-type rotation, effected by vibration about the axis of the container of the fluid or pulsation of the container boundary, provided that $g_{1}=-K_{s}^{2}$ and $g_{2}=-B K_{s}$. 
The solutions can be expressed in terms of the solutions of the standard Mathieu equation

$$
y^{\prime \prime}+\left(a_{1}-2 q \cos 2 z\right) y=0 .
$$

These Mathieu functions traditionally enter fluid mechanics in connection with the sloshing of water in a flat-bottomed elliptical tub. They also appear in mechanical, electrical, and electromagnetic systems (McLachlan [7]). The variety of types of Mathieu functions is daunting - 10 unmodified types and 10 modified (solutions of $\left.y^{\prime \prime}-\left(a_{1}-2 q \cosh 2 z\right) y=0\right)$. For the unmodified case, of interest here, 8 of the types are stable-

$$
\begin{aligned}
& \mathrm{ce}_{m}(z, q), \quad \mathrm{se}_{m}(z, q), \quad \mathrm{ce}_{m+\beta}(z, q), \\
& \mathrm{fe}_{m}(z, q), \quad \mathrm{ge}_{m}(z, q), \quad \mathrm{se}_{m+\beta}(z, q), \\
& \mathrm{fek}_{m}(z,-q), \quad \operatorname{gek}_{m}(z,-q)
\end{aligned}
$$

- and two are unstable $-\operatorname{ceu}_{m+\mu}(z, q)$ and $\operatorname{ceu}_{m+\mu}(-z, q)$. In this MacLachlan classification, 'unstable' means unbounded as $z \rightarrow+\infty$, and $m$ is a positive integer.

The instability results from initial conditions which yield an admixture of stable and unstable types, as will usually occur unless $\left(q, a_{1}\right)$ lies in a stability region. These resemble a stack of infinitely long moustaches tending to zero width along asymptotes, and increasing in height and width with increasing $a_{1}$ and $q$. For $a_{1}<.04 q^{2}$ the stability regions become quite slender, and for $a_{1}>2.5 q^{2}$, the instability regions become quite slender. In between those two parabolas, the instability and stability zones are of about equal size. The first stability region includes the origin in $\left(q, a_{1}\right)$ space.

When $G(t)=q_{1}+h_{1} \cos \nu t$ with $q_{1}$ and $h_{1}$ real, (28) becomes

$$
\ddot{A}+B S \dot{A}=S A\left(g_{1}+h_{1} \cos \nu t\right) \text {. }
$$

On taking $\nu t=r, A(t)=u(r)$, we find

$$
u^{\prime \prime}(r)+(B S / \nu) u^{\prime}(r)=\left(S / \nu^{2}\right)\left(q_{1}+h_{1} \cos r\right) u(r) .
$$

Now let

$$
K=B S / \nu, \quad c=S g_{1} / \nu^{2}, \quad d=S h_{1} / \nu^{2}
$$

so that

$$
u^{\prime \prime}+K u^{\prime}=(c+d \cos r) u .
$$

The substitution $u=e^{-K r / 2} v$ yields

$$
v^{\prime \prime}+\left(-c-K^{2} / 4-d \cos r\right) v=0 .
$$

Assuming $|d| \ll 1$ and $|K|=O(|d|)$, a refined first-stable-zone estimate for $u$ (Jordan and Smith [5], Section 8.7) requires that $(-c-1 / 4)^{2}>\left(d^{2}-K^{2}\right) / 4$. 
Since $c=S g_{1} / \nu^{2}, d=S h_{1} / \nu^{2}, K=B S / \nu$, the inequality becomes

$$
S^{2}\left(g_{1}^{2}-h_{1}^{2} / 4\right)+\nu^{2}\left(S g_{1} / 2+B^{2} S^{2} / 4\right)+\nu^{4} / 16>0 .
$$

This is certainly satisfied for large $\left|g_{1}\right|$ or large $\nu$, for fixed $h_{1}, S$ and $B$.

A rough envelope of the higher stability zones is provided by $a_{1}=-c-K^{2} / 4$ $>.04(2 d)^{2}$, or $-S g_{1}-(B S)^{2} / 4>.16\left(S h_{1} / \nu\right)^{2}$. Since Charwat's 'more practical case' requires $S K_{s}^{2}<(D S)^{2} / 4$, and sôlid rótátion implies $g_{1} \sim-K_{s}^{2}$, it foliows from these conditions that $-S g_{1}-(B S)^{2} / 4<0$, so that the motion does not lie in the rough stability envelope. Each stability zone has a long tail not included in the rough envelope, so that stability cannot really be excluded without a detailed examination. However, it is plausible that most combinations of $S, g, h, B, K_{s}$ and $\nu$ will result in instability and irregular tracer motions under periodic parametric perturbations.

\section{Conclusion}

The asymptotic results both for the solid body and free vortex cases are physically obvious: particles more dense than the fluid will be forced outward. Coriolis forces induce the spiralling. The transient damped scalloping with a fixed scalloping frequency is a little surprising, but resembles some minor rotating oscillatory phenomena found elsewhere in fluid mechanics and ionospheric charged particle motions. It may be mathematically related to the ropy motions observed in the recent fly-by of the rings of Saturn. The instability under sinusoidal 'parametric' perturbation (except for high frequencies, etc.) is hardly surprising.

\section{Acknowledgement}

The author wishes to thank Professor James Vanyo of the UCSB Department of Mechanical and Environmental Engineering for valuable comments.

\section{References}

[1] A. Charwat, "Motion of near-neutrally buoyant tracers in vortical nlows", Phys. Flutds 20 (1977), 401-403.

[2] R. H. Fowler, "Emden's and similar differential equations", Quart. J. Math. Oxford 92 (1931), 259-290. 
[3] H. S. Green and R. B. Leipnik, "Diffusion and conductivity of plasmas in strong external fields", Internat. J. Engrg. Sci. 3 (1965), 491-514.

[4] E. L. Ince, Ordinary differential equations (Dover, New York, 1944; Longmans Green, London, 1926).

[5] D. W. Jordan and P. Smith, Nonlinear ordinary differential equations (Oxford University Press, Oxford, 1977).

[6] R. B. Leipnik, P. W. Seymour and A. F. Nicholson, "Charged particle motion in a time-dependent axially symmetric field", A ustral. J. Phys. 18 (1965), 553-566.

[7] N. W. McLachlan, Theory and application of Mathieu functions (Oxford University Press, Oxford, 1947). 